\title{
Maxwell-Bloch approach to excess quantum noise
}

\author{
S. M. Dutra, K. Joosten, G. Nienhuis, N. J. van Druten, A. M. van der Lee, M. P. van Exter, and J. P. Woerdman \\ Huygens Laboratory, University of Leiden, P.O. Box 9504, 2300 RA Leiden, The Netherlands
}

(Received 9 December 1998)

\begin{abstract}
To meet recent experimental advances, we generalize the intuitively appealing nonorthogonal-mode theory of excess quantum noise by introducing a Maxwell-Bloch description of the gain medium. The resulting equations extend the nonorthogonal-mode approach beyond the class A linear-gain regime providing a general starting point for theoretical descriptions of excess quantum noise. As an illustration of our theory, we derive rate equations describing excess quantum noise in class B lasers and obtain the non-Lorentzian spectrum due to the coloring of excess noise in class A lasers accounting for gain saturation. [S1050-2947(99)03106-6]
\end{abstract}

PACS number(s): 42.50.Lc, 42.55.-f, 42.60.Da

Excess quantum noise is an intriguing effect that has been demonstrated recently in several types of lasers [1-3]. Since Petermann first predicted it [4], much effort was put in trying to understand this effect [5-7]. In 1989, Siegman proposed a semiclassical theory that derives excess noise as a universal consequence of mode nonorthogonality [6,7]. In addition to providing experimentalists with an appealing semiclassical picture of excess noise, this "geometrical" [8] theory is also a powerful calculational tool. This theory was developed for class A lasers [9], where the atomic variables relax much faster than the field, within the linear isotropic gain approximation [7]. However, the presence of relaxation oscillations in the lasers $\left(\mathrm{HeXe}\right.$ and $\left.\mathrm{Nd}^{3+}: \mathrm{YVO}_{4}\right)$ where excess noise was observed so far [1-3] shows that none of them are strictly class A [10]. Moreover, although the "geometrical", theory derives excess noise as white noise, it was recently found to be colored [11]. Here, we show how the " geometrical" theory can be extended to meet these new experimental challenges [12].

The approach taken here parallels that of ordinary laser theory where the combined dynamics of electric field and atomic variables is generally described by Maxwell-Bloch equations [13]. We incorporate the formalism of biorthogonal modes into these Maxwell-Bloch equations. After describing the theory we will illustrate it with two applications: an example of a nonclass A laser, and a class A laser where both gain saturation and the dynamics of nonlasing modes are taken into account. The latter case will give rise to the recently discovered phenomenon of the coloring of excess noise [11].

The microscopic model we adopt consists of a system of homogeneously broadened two-level atoms [14] of resonance frequency $\omega_{a}$ and dipole strength $\mu_{a}$ embedded in a dielectric host of refractive index $n_{r}$ and interacting with the quantized electromagnetic field in a cavity. The cavity is a single-ended output laser cavity as in Ref. [7] and we adopt the same notation and normalization conventions used there for the cavity biorthogonal modes. The atoms are also coupled to reservoirs yielding the decay rates $\gamma_{\|}$for the inversion and $\gamma_{\perp}$ for the polarization together with their associated noise fluctuations. To avoid the complications of expanding quantized fields into nonorthogonal cavity modes [15-17], we reduce the quantum Langevin equations to equivalent $c$-number Langevin equations and expand the $c$-number counterparts of the quantum-mechanical operators instead of the operators themselves. The equivalent $c$-number Langevin equations are obtained by choosing the normal ordering and neglecting thermal noise in the field as in Ref. [18]. This procedure retains quantum correlations but only up to second moments of the dynamical variables [18]. Assuming that spatial and spectral hole burning can be neglected [19] and that the pumping is spatially uniform, we obtain the following Maxwell-Bloch equations for lasers with nonorthogonal modes:

$$
\dot{c}_{q n}(t)=\left\{i \omega_{q n}+\frac{c \ln \left|\tilde{\gamma}_{n}\right|}{n_{r} p}\right\} c_{q n}(t)-i \frac{c^{2}}{2 n_{r}^{2}} \omega_{c} \mu_{0} p_{q n}(t),
$$

$\dot{p}_{q n}(t)=\left\{i\left(\omega_{a}-\omega_{c}\right)-\gamma_{\perp}\right\} p_{q n}(t)+i \frac{\mu_{a}^{2}}{\hbar V} \mathcal{D}(t) c_{q n}(t)+F_{q n}(t)$,

$$
\begin{aligned}
& \dot{\mathcal{D}}(t)=-\gamma_{\|} \mathcal{D}(t)+\Lambda_{0}+\frac{i}{2 \hbar} \sum_{n q n^{\prime} q^{\prime}} T_{n q}^{n^{\prime} q^{\prime}}\left\{p_{q n}(t) c_{q^{\prime} n^{\prime}}^{*}(t)\right. \\
& \left.-p_{q^{\prime} n^{\prime}}^{*}(t) c_{q n}(t)\right\}+F_{\mathcal{D}}(t) \text {, }
\end{aligned}
$$

where $c_{q n}(t)$ and $p_{q n}(t)$ are the expansion coefficients of the slowly varying electric field (with the laser central frequency $\omega_{c}=\beta_{c} c / n_{r}$ separated out) and polarization, respectively, in the paraxial approximation corresponding to the $q$ th longitudinal mode and the $n$th transverse mode. Here $c$ is the speed of light in vacuum and our $c_{q n}(t)$ and $p_{q n}(t)$ correspond to $\sqrt{p} \widetilde{c}_{q n}(t) \exp \left(i \omega_{q n} t\right)$ and $\sqrt{p} \tilde{p}_{q n}(t) \exp \left(i \omega_{q n} t\right)$, respectively, in Refs. [6,7], with $p$ being the cavity round-trip length and the mode frequencies $\omega_{q n}$ being relative to the central frequency $\omega_{c}$. The other symbols stand for the following: $\mathcal{D}(t)$ $=\mathcal{N}_{2}(t)-\mathcal{N}_{1}(t)$ is the spatially uniform inversion, $\mathcal{N}_{j}$ is the atomic population in level $j, \Lambda_{0}$ is the pumping rate, $V$ is the cavity volume, and the dimensionless "weighted overlap factor', $T_{n q}^{n^{\prime} q^{\prime}} \equiv \int d^{3} r \Gamma_{q q^{\prime}}^{z / p}\left(\tilde{\gamma}_{n^{\prime}}^{*} \tilde{\gamma}_{n}\right)^{(p-z) / p} \tilde{u}_{n} \tilde{u}_{n^{\prime}}^{*} / p$, with $\tilde{u}_{n}(\mathbf{r})$ being the $n$th transverse mode, $\tilde{\gamma}_{n}$ the corresponding complex eigenvalue, $\Gamma_{q q^{\prime}} \equiv \exp \left\{i\left(q^{\prime}-q\right) 2 \pi\right\}$, and the integration being over the cavity volume. The Langevin forces are fully defined by their second-order moments $\left\langle F_{i}(t) F_{j}\left(t^{\prime}\right)\right\rangle$ 
$=2 D_{i}^{j} \delta\left(t-t^{\prime}\right)$ and $\left\langle F_{i}(t) F_{j}^{*}\left(t^{\prime}\right)\right\rangle=2 \bar{D}_{i}^{j} \delta\left(t-t^{\prime}\right)$ with all other moments vanishing [13]. The diffusion coefficients $D_{i}^{j}$ and $\bar{D}_{i}^{j}$ are given by

$$
\begin{gathered}
\bar{D}_{q n}^{q^{\prime} n^{\prime}}=\frac{4 \mu_{a}^{2} \gamma_{\perp}}{V}\left\{\mathcal{N}_{2}-\frac{\gamma_{\|}}{4 \gamma_{\perp}}\left[\mathcal{D}-\frac{\Lambda_{0}}{\gamma_{\|}}\right]\right\} \frac{T_{n q}^{n^{\prime} q^{\prime}} K_{n^{\prime} n}^{T}}{\tilde{\gamma}_{n} \tilde{\gamma}_{n^{\prime}}^{*} \Gamma_{q q^{\prime}}}, \\
D_{q n}^{q^{\prime} n^{\prime}}=i \frac{\mu_{a}^{2}}{\hbar V} \sum_{k m l s} T_{n q n^{\prime} q^{\prime}}^{m k s s} c_{k m} p_{l s}, \\
D_{q n}^{\mathcal{D}}=-\frac{\gamma_{\|}}{2}\left\{1+\frac{\Lambda_{0}}{\left.\gamma_{\|} \mathcal{N}\right\}} p_{q n},\right. \\
D_{\mathcal{D}}^{\mathcal{D}}=\frac{\gamma_{\|}}{2}\left\{\mathcal{N}-\frac{\Lambda_{0} \mathcal{D}}{\gamma_{\|} \mathcal{N}}\right\}+\frac{i}{2 \hbar} \sum_{n q n^{\prime} q^{\prime}} T_{n q^{n^{\prime} q^{\prime}}} p_{q n^{n}} c_{q^{\prime} n^{\prime}}^{*}+\text { c.c. },
\end{gathered}
$$

where the tensorial overlap factor in Eq. (2b) is given by $T_{n q n^{\prime} q^{\prime}}^{m k s l} \equiv\left(V / p^{2}\right) \int d^{3} r \Pi_{n q n^{\prime} q^{\prime}}^{m k s l} \widetilde{\phi}_{n} \widetilde{u}_{m} \widetilde{\phi}_{n^{\prime}} \tilde{u}_{s}, \mathcal{N}=\mathcal{N}_{2}+\mathcal{N}_{1}$ is the total number of atoms, and $K_{n^{\prime} n}^{T} \equiv \eta_{n n^{\prime}}^{-1} \int d^{2} s \widetilde{\phi}_{n^{\prime}}^{*} \widetilde{\phi}_{n}$ in Eq. (2a) is the transverse Petermann factor between mode $n^{\prime}$ and $n$, with $\mathbf{s}$ being a position on the transverse reference plane and $\widetilde{\phi}_{n}(\mathbf{r})$ the $n$th adjoint mode [6,7]. The abbreviations above stand for: $\Pi_{n q n^{\prime} q^{\prime}}^{m k s l}(z)$ $\equiv\left(\Gamma_{k q} \Gamma_{l q^{\prime}}\right)^{(z / p)}\left(\tilde{\gamma}_{n} \tilde{\gamma}_{n^{\prime}} \tilde{\gamma}_{m}^{-1} \tilde{\gamma}_{s}^{-1}\right)^{(z-p) / p} \quad$ and $\quad \eta_{n n^{\prime}}$ $\equiv \int d^{2} s \tilde{u}_{n} \tilde{u}_{n^{\prime}}^{*}$ is the "transverse overlap factor."

To test our general theory, we will now show that for class A lasers in the linear isotropic gain approximation, we recover Siegman's equations for the field-expansion coefficients [7]. In this case, we adiabatically eliminate $\tilde{p}_{q n}(t)$ $\equiv p_{q n}(t) \exp \left(-i \omega_{q n} t\right) / \sqrt{p}$ and $\mathcal{D}(t)$ in Eqs. (1) neglecting saturation. Then we obtain the following single equation of motion for the field:

$$
\dot{\tilde{c}}_{q n}(t)=\frac{c}{n_{r}}\left\{\alpha+\frac{\ln \left|\tilde{\gamma}_{n}\right|}{p}\right\} \tilde{c}_{q n}(t)+\tilde{p}_{q n}^{N}(t),
$$

where $\alpha=\mu_{0} \mu_{a}^{2} c \omega_{c} \Lambda_{0} /\left(2 n_{r} \hbar V \gamma_{\perp} \gamma_{\|}\right)$is the linear gain and the noise $\tilde{p}_{q n}^{N}(t)=-i c^{2} \omega_{c} \mu_{0} \exp \left(-i \omega_{q n} t\right) F_{q n}(t) /\left(2 n_{r}^{2} \gamma_{\perp}\right)$ has the following correlation function derived from Eq. (2a):

$$
\left\langle\tilde{p}_{q n}^{N}(t) \tilde{p}_{q n}^{N *}\left(t^{\prime}\right)\right\rangle=2 K_{n n}^{T} \hbar \alpha \frac{\mathcal{N}_{2} c^{3} \omega_{c} \mu_{0}}{\mathcal{D} n_{r}^{3}} \frac{\tilde{\gamma}_{n}^{2}-1}{p \tilde{\gamma}_{n}^{2} \ln \left|\tilde{\gamma}_{n}\right|} \delta\left(t-t^{\prime}\right) .
$$

Thus, we have recovered the result of Ref. [7] with the noise polarization correlation function (4) derived now from the Maxwell-Bloch noise correlation function (2a), whereas Ref. [7] obtains it by a heuristic argument.

Equations (1) describe excess quantum noise in any laser where the inversion can be assumed not to depend on position. It is interesting to reduce Eqs. (1) to simple cases other than class A. As a first illustration of our general theory, let us apply it to class B lasers [9]. In such a laser the polarization can be adiabatically eliminated but not the inversion [9]. For simplicity, we will also assume that all the nonlasing modes are strongly damped (this assumption will be dropped when we discuss the coloring of excess noise). We end up with the following rate equations for the average number of photons in the laser $s(t) \equiv T_{n q}^{n q} n_{r}^{2}\left|\tilde{c}_{q n}(t)\right|^{2} /\left(2 \hbar \omega_{c} c^{2} \mu_{0}\right)$ and the inversion $\mathcal{D}(t)$,

$$
\begin{gathered}
\dot{s}=\left\{\gamma_{\|} \beta \mathcal{D}+2 \frac{c \ln \left|\tilde{\gamma}_{n}\right|}{n_{r} p}\right\} s+K R_{s p}+f, \\
\dot{\mathcal{D}}=\Lambda_{0}-\gamma_{\|} \mathcal{D}\{1+2 \beta s\}-2 K R_{s p}-2 f+f_{\mathcal{D}} .
\end{gathered}
$$

The factors of 2 appearing in the inversion Eq. (5b) are a consequence of our two-level description [14], $\beta$ $\equiv c^{2} \omega_{c} \mu_{a}^{2} \mu_{0} /\left(n_{r}^{2} \hbar \gamma_{\perp} \gamma_{\|} V\right)$ is the spontaneous emission factor [20], and $R_{s p} \equiv \gamma_{\|} \beta \mathcal{N}_{2}$ is the ordinary spontaneous emission contribution to the laser field, which is enhanced in Eqs. (5) by the excess noise factor $K$ for the lasing mode. The Langevin force $f(t)$ describes the spontaneous emission noise, with $\left\langle f(t) f\left(t^{\prime}\right)\right\rangle=2 K R_{s p} s \delta\left(t-t^{\prime}\right)$. The other Langevin force in Eq. $(5 \mathrm{~b}), f_{\mathcal{D}}(t)$, describes the inversion noise which, in this regime $\left(\gamma_{\perp} \gg \gamma_{\|}\right)$, is not correlated to $f(t)$ but only to itself, $\left\langle f_{\mathcal{D}}(t) f_{\mathcal{D}}\left(t^{\prime}\right)\right\rangle=2 D \delta\left(t-t^{\prime}\right)$, where $D=\gamma_{\|}\{\mathcal{N}$ $\left.-\left[\Lambda_{0} \mathcal{D} /\left(\gamma_{\|} \mathcal{N}\right)\right]-2 \beta s \mathcal{D}\right\}$.

Rate equations similar to Eqs. (5) have been postulated before to describe bad-cavity lasers [see Eq. (1) of Ref. [21]]. A bad-cavity laser is a laser where $\gamma_{\perp}^{-1}$ is not small compared to the lifetime of a photon in the cavity. To compare our rate equations (5) with those used in Ref. [21], we notice that the cavity loss rate $-c \ln \left|\tilde{\gamma}_{n}\right| /\left(n_{r} p\right)$ has to be replaced by a "dressed" cavity loss rate dependent on the inversion [22]. Then Eqs. (5) coincide with the rate equations used in Ref. [21] apart from the last three terms on the righthand side of the equation of motion for the inversion (5b) describing the spontaneous emission depletion of the inversion $\left(-2 K R_{s p}\right)$ and the two Langevin forces. Inversion noise is often neglected in the literature $[17,22,23]$ but it was suggested that it can become important in class B lasers [24]. To the best of our knowledge, the total noise in the inversion described by the last terms on the right-hand side of Eq. (5b) has not been derived before for class B lasers with both transverse and longitudinal excess quantum noise.

It has been discovered recently that excess noise is not just a geometrical effect of mode nonorthogonality. The dynamical evolution of the noise-driven nonlasing modes also plays a role in the generation of excess noise. The signature of this dynamical contribution is the coloring of excess noise recently demonstrated in an experiment [11]. As a second illustration of our theory, we use the Maxwell-Bloch approach presented here to calculate the optical spectrum of the laser and demonstrate this coloring as a deviation from the normal Lorentzian spectrum [11]. We do so by reducing the Maxwell-Bloch Eqs. (1) to a Lamb third-order equation for the electric field, this time taking into account the nonlasing modes, unlike in Eqs. (5). For simplicity, we consider the case where only one longitudinal mode, e.g., $q=1$, is relevant (short cavity) and every transverse mode experiences the same gain $\left(\omega_{a}-\omega_{c}-\omega_{1 n} \ll \gamma_{\perp}\right.$ for every $\left.n\right)$; the lasing mode being the one with the smallest cavity loss rate. We calculate the spectrum using the Wiener-Khintchine theorem and by linearizing the equations around the steady state. Then the lasing mode amplitude is expressed as $c_{L}(t)=\{r$ 
$+\delta r(t)\} \exp \{i \phi(t)\}$, where $r^{2}$ is the stabilized laser intensity, $\delta r$ represents an in-phase fluctuation in the lasing mode amplitude (which determines the intensity fluctuations), and $\phi$ is the phase of the lasing mode. The coloring arises from time correlations such as $\left\langle\exp \{i \phi(t)\} c_{n}^{*}(t+\tau)\right\rangle$ with $n \neq L$ that, due to mode nonorthogonality, also have to be accounted for in addition to the ordinary time correlation $\langle\exp \{i[\phi(t)-\phi(t$ $+\tau)]\}$, which is alone responsible for the laser linewidth in lasers with orthogonal modes. Our calculation yields the following expression for the laser spectrum outside the cavity at the position of the exit mirror,

$$
\begin{aligned}
S(\omega)= & \frac{\left|t_{L}\right|^{2} D_{L L} / \pi}{\left(\omega-\omega_{L}\right)^{2}+D_{L L}^{2} / r^{4}} \\
& \times\left\{1+2 \sum_{n \neq L} \operatorname{Re}\left(\left[1-\frac{\gamma_{n L}+i \Delta_{n L}}{\gamma_{n L}-i\left(\omega-\omega_{n}\right)}\right]\right.\right. \\
& \left.\left.\times \frac{t_{L} t_{n}^{*} \eta_{L n}}{\left|t_{L}\right|^{2}} \frac{D_{L n}}{D_{L L}}\right)\right\},
\end{aligned}
$$

where $t_{j}$ are the amplitude transmissivities of the cavity output mirror, $\gamma_{n L} \equiv c \ln \left|\tilde{\gamma}_{L} / \tilde{\gamma}_{n}\right| /\left(n_{r} p\right)$ is the difference between the "cold" cavity damping of the $n$th nonlasing mode and the lasing mode (it specifies how much below threshold every nonlasing mode is), $\Delta_{n L}$ is the detuning between the $n$th nonlasing-mode frequency $\omega_{n}$ and the lasing-mode frequency $\omega_{L}, D_{L L} / r^{2}$ is the excess-noise-enhanced linewidth of the lasing mode alone, and $2 D_{L n} \equiv i\left(c^{2} \omega_{c} \mu_{0} /\right.$ $\left.2 n_{r}^{2} \gamma_{\perp}\right)^{2} \bar{D}_{1 n}^{1 L}$.

We notice that if the net loss rates of the nonlasing modes are much larger than the $K$-enhanced laser linewidth there is no coloring and the spectrum is simply given by the first Lorentzian on the right-hand side of Eq. (6). In Fig. 1, we plot the spectrum for the case where all but one of the nonlasing modes have a loss rate much larger than the ordinary $K$-enhanced laser linewidth, so that only one nonlasing mode contributes to the line shape. In Fig. 1(a), the net loss rate $\gamma_{n L}$ of the nonlasing mode has been chosen as ten times the ordinary $K$-enhanced laser linewidth $D_{L L} / r^{2}$. Then deviations from the normal Lorentzian spectrum only start appearing as one moves towards the wings of the spectrum [Fig. 1(a) is in logarithmic scale] in agreement with the timedomain argument [11]: large frequencies mean small times before the fluctuations in the nonlasing mode become completely damped. One way to bring these deviations closer to the central part of the spectrum is to increase the cavity lifetime of the nonlasing mode. In fact, as we can see from Fig. 1(b) where we have decreased $\gamma_{n L}$ by a factor of 50, deviations from the Lorentzian shape become visible even in a normal linear scale in the central part of the spectrum. We would like to stress that the spectrum seen in Fig. 1(b) is not
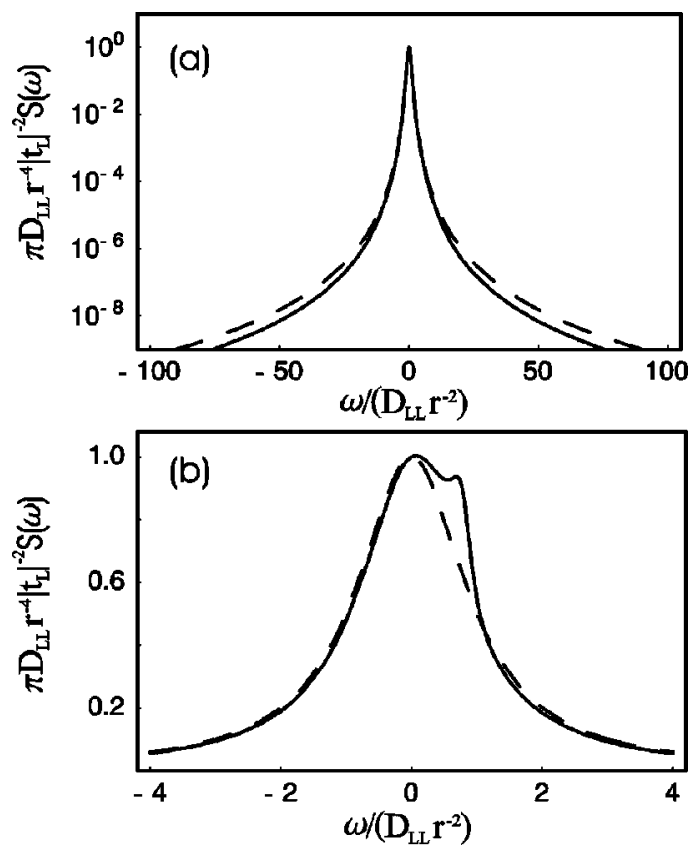

FIG. 1. In (a), we plot the spectrum (full line) in a log scale for $\gamma_{n L}=10$ and $\Delta_{n L}=1$. In (b), we plot the spectrum in a linear scale for $\gamma_{n L}=0.2$ and $\Delta_{n L}=0.8$. All rates are in units of $D_{L L} / r^{2}$. We also plot in the same figures the Lorentzian spectrum for the laser mode alone (dotted line) that one would obtain if the coloring were negligible.

a double-peak spectrum of the usual sort that is associated with the superposition of two modes. The close-to-threshold nonlasing mode considered here is still below threshold and is not lasing. For the numerical values used in the plot of Fig. 1(a), the intensity of the nonlasing mode is about 100 times weaker than that of the lasing mode, and for those of Fig. 1(b), 10 times weaker. So in a laser resonator where these modes were orthogonal the nonlasing mode would not be visible in the spectrum as in these plots. Moreover, as can be seen from Eq. (6), the spectrum is not a sum of Lorentzians as in the case of two lasing modes but rather a product of Lorentzians as in Ref. [11].

To conclude, we have presented a unifying framework for excess noise, based on a Maxwell-Bloch approach, from which previous theoretical descriptions can be derived in a consistent way. We have shown how this theoretical framework can be used to derive corrections to previous descriptions and also to explain phenomena such as the recently discovered coloring of excess noise [11].

S.M.D. would like to thank J. Steinberg for an interesting discussion. This work is part of the research program of the "Stichting voor Fundamenteel Onderzoek der Materie" (FOM), the European Union ESPRIT Project No. 20029 (ACQUIRE), and the TMR network No. ERB4061 PL951021 (Microlasers and Cavity QED). The research of N. J. van Druten has been made possible by the "Koninklijke Nederlandse Akademie van Wetenschappen." 
[1] Y.-J. Cheng, P. L. Mussche, and A. E. Siegman, IEEE J. Quantum Electron. QE-30, 1498 (1994); Y.-J. Cheng, G. Fanning, and A. E. Siegman, Phys. Rev. Lett. 77, 627 (1996).

[2] M. A. van Eijkelenborg, A. M. Lindberg, M. S. Thijssen, and J. P. Woerdman, Phys. Rev. Lett. 77, 4314 (1996); Opt. Commun. 137, 303 (1997); A. M. Lindberg, M. A. van Eijkelenborg, K. Joosten, G. Nienhuis, and J. P. Woerdman, Phys. Rev. A 57, 3036 (1998).

[3] O. Emile, M. Brunel, A. Le Floch, and F. Bretenaker, Europhys. Lett. 43, 153 (1998).

[4] K. Petermann, IEEE J. Quantum Electron. QE-15, 566 (1979).

[5] K. Ujihara, IEEE J. Quantum Electron. QE-20, 814 (1984); H. A. Haus and S. Kawakami, ibid. QE-21, 63 (1985); P. Goldberg et al., Phys. Rev. A 44, 1969 (1991); 44, 4556 (1991); J. Mod. Opt. 38, 1421 (1991).

[6] A. E. Siegman, Phys. Rev. A 39, 1253 (1989).

[7] A. E. Siegman, Phys. Rev. A 39, 1264 (1989).

[8] Geometrical in the sense that this theory derives excess noise as a consequence of mode nonorthogonality.

[9] F. T. Arecchi, Nucl. Phys. B 2, 13 (1987).

[10] It remains to be seen to what extent these nonclass A features affect the validity of Siegman's theory. But only a generalization of the geometrical theory to lasers that are not class A can provide a systematic theoretical basis for such a discussion.

[11] A. M. van der Lee, M. P. van Exter, A. L. Mieremet, N. J. van Druten, and J. P. Woerdman, Phys. Rev. Lett. 81, 5121 (1998).

[12] Other generalizations of the "geometrical" theory of Refs. $[6,7]$ proposed so far have remained within a linear class A description. That is the case of the quantum versions of the "geometrical" theory in Refs. $[15,16]$ and of a recent generalization to any linear system [A. Siegman (private communication)].

[13] Here we use the name Maxwell-Bloch for the Langevin equa- tions of motion for the field, the polarization, and the inversion where noise forces are included as in H. Haken, in Laser Theory (Springer-Verlag, Berlin, 1983).

[14] We adopt a three-level laser model where the top level used in the pumping scheme has been adiabatically eliminated leading to the effective pumping rate density $\Lambda_{0}$. As the lower state is also the ground state here, the total number of atoms in the pair of levels involved in the laser is conserved.

[15] I. H. Deutsch, J. C. Garrison, and E. M. Wright, J. Opt. Soc. Am. B 8, 1244 (1991).

[16] Ph. Grangier and J.-Ph. Poizat, Eur. Phys. J. D 1, 97 (1998); (unpublished).

[17] S. Prasad, Phys. Rev. A 46, 1540 (1992).

[18] C. Benkert, M. O. Scully, J. Bergou, L. Davidovich, M. Hillery, and M. Orszag, Phys. Rev. A 41, 2756 (1990); M. I. Kolobov, L. Davidovich, E. Giacobino, and C. Fabre, ibid. 47, 1431 (1993).

[19] The neglect of spatial hole burning is quite reasonable in HeXe lasers, because in gas lasers the rapid thermal motion of the atoms prevents hole burning, but not in solid state lasers such as $\mathrm{Nd}^{3+}: \mathrm{YVO}_{4}$. The neglect of spectral hole burning is reasonable for both $\mathrm{HeXe}$ and $\mathrm{Nd}^{3+}: \mathrm{YVO}_{4}$ lasers.

[20] P. R. Rice and H. J. Carmichael, Phys. Rev. A 50, 4318 (1994); M. P. van Exter, G. Nienhuis, and J. P. Woerdman, ibid. 54, 3553 (1996).

[21] M. A. van Eijkelenborg, M. P. van Exter, and J. P. Woerdman, Phys. Rev. A 57, 571 (1998).

[22] S. J. M. Kuppens, M. P. van Exter, and J. P. Woerdman, Phys. Rev. Lett. 72, 3815 (1994).

[23] M. P. van Exter, S. J. M. Kuppens, and J. P. Woerdman, Phys. Rev. A 51, 809 (1995).

[24] P. Paoli, A. Politi, and F. T. Arecchi, Z. Phys. B 71, 403 (1988). 